\title{
Synthesis and Thermoelectric Property of 1D Flexible PEDOT: p-TSA/Glass Fiber
}

\author{
Xiaohua Chen 1 , Guangshi Tang1* ${ }^{*}$ Junqing Pan ${ }^{1}$, Hanfu Wang ${ }^{2}$ \\ ${ }^{1}$ College of Science, Beijing University of Chemical Technology, Beijing, China \\ ${ }^{2}$ CAS Key Laboratory of Nanosystem and Hierarchical Fabrication, National Center for Nanoscience and Technology of China, \\ Beijing, China \\ Email: *tgs1803@163.com
}

How to cite this paper: Chen, X.H., Tang, G.S., Pan, J.Q. and Wang, H.F. (2018) Synthesis and Thermoelectric Property of 1D Flexible PEDOT: p-TSA/Glass Fiber. Journal of Minerals and Materials Characterization and Engineering, 6, 448-463.

https://doi.org/10.4236/jmmce.2018.63032

Received: April 25, 2018

Accepted: May 27, 2018

Published: May 30, 2018

Copyright $(9) 2018$ by authors and Scientific Research Publishing Inc. This work is licensed under the Creative Commons Attribution International License (CC BY 4.0).

http://creativecommons.org/licenses/by/4.0/ Open Access

\begin{abstract}
Exploiting the thermal insulation properties of glass fiber and excellent conductivity of conducting polymer, a novel one-dimensional (1D) composite thermoelectric material, based on poly(3,4-ethylenedioxythiophene): p-toluenesulfonic acid (PEDOT: p-TSA)/glass fiber, is prepared by coating the PEDOT: p-TSA on the surface of glass fiber with in situ polymerization method. We hope the materials can bring out the performance of the "electron conductor, photon glass". During the polymerization process, the effects of oxidant concentration and dopant mass fraction on thermoelectric properties of the materials are investigated. The group type of the polymer chain and the morphology of the samples were characterized by Fourier transform infrared spectroscopy (FT-IR) and scanning electron microscopy (SEM), respectively. The maximal Seebeck coefficient $(S)$ and electric conductivity $(\sigma)$ of the pristine sample are $32 \mu \mathrm{VK}^{-1}$ and $169 \mathrm{Sm}^{-1}$, respectively. After further post-processing with methanol, the thermoelectric properties of materials were improved, and the maximum value of $S$ and $\sigma$ increased greatly to 48.5 $\mu \mathrm{VK}^{-1}$ and $3184 \mathrm{Sm}^{-1}$, respectively. The maximal power factor $(P F)$ of materials also increased from $0.12 \mu \mathrm{Wm}^{-1} \mathrm{~K}^{-2}$ to $6.74 \mu \mathrm{Wm}^{-1} \mathrm{~K}^{-2}$. Moreover, we have proposed a preliminary explanation on the carrier transport mechanism.
\end{abstract}

\section{Keywords}

Thermoelectric Materials, Flexible Fiber, Seebeck Coefficient, Organic-Inorganic Composite, In Situ Polymerization

\section{Introduction}

Thermoelectric materials have attracted increasing attention, which are envi- 
ronmentally friendly and hold great promise for improving energy waste, due to the intensification of energy and environment crisis [1] [2] [3]. The performance of thermoelectric (TE) materials is generally evaluated by the dimensionless figure of merit, $Z T=S^{2} \sigma T / \kappa$, where $S$ is the Seebeck coefficient, $\sigma$ and $\kappa$ are the electrical and thermal conductivity, respectively. As the $\kappa$ of some samples is difficult to measure accurately, the $P F, P F=S^{2} \sigma$, can be used to estimate the thermoelectric performance. The quantum wire of the $1 \mathrm{D}$ thermoelectric materials could further improve the density of state (DOS) near the Fermi level than the quantum wells [4]. The increase of DOS results in a corresponding increase in the amount of the effective carriers, so the value of $\mathrm{S}$ and the $Z T$ of materials would increase. As a result, the $Z T$ of one-dimensional thermoelectric materials may be larger than that of the bulk and two-dimensional thin film. At present, the maximal $Z T$ of PEDOT: PSS film of p-type and poly[Kx (Ni-ett)] of n-type thermoelectric materials are 0.42 [5] and 0.2 [6], respectively. The maximal $Z T$ of PEDOT-Tos film is 0.25 [7]. However, the maximum of $Z T$ for 1D thermoelectric materials is not definite.

The first principle is widely used in the theoretical calculation of the structure and thermoelectric properties of the inorganic semiconductor model [8] [9]. Wang, et al., calculated the structure, electron and thermoelectric properties of InSe nanotubes (InSeNTs) using the first principle based on density functional theory (DFT) [8]. The results of calculations indicated that $(2,2)$ nanotubes obtained a higher $Z T$ than that of other studied nanotubes whatever they were semiconductors or metals. For organic semiconductors, there are a few theoretical studies that can be used to predict the thermoelectric performance at present. Some researchers studied the effects of physical parameters, such as degree of polymerization, crystallinity of polymer, and microscopic structure of the organic materials [10] [11] [12]. Yang et al. [10] studied the thermoelectric properties of the quasi-one-dimensional (Q1D) self-assembled molecular nanowires (NWs), based on the rigorous Kubo formula using the Holstein model. The thermoelectric properties depended on a variety of physical parameters including intersite coupling, electron-phonon interaction, and so on. Their calculation showed that the higher value of $Z T$ was about 15.2, assuming that the phonon thermal conductivity of the molecular NWs that is composed of one-dimensional conducting polymer chains was $0.2 \mathrm{Wm}^{-1} \mathrm{~K}^{-1}$. It was much greater than the maximum value of $Z T$ obtained from the inorganic thermoelectric materials so far. The Holstein model was also used to study the thermoelectric properties of one-dimensional polymer chains, such as P3HT and PEDOT: PSS. Although the causal relationship between the microstructure and properties of the materials is still far from clear, the design of materials can refer to the results of theoretical calculation a little. The theoretical study indicated that low-dimensional conductive polymer was expected to become thermoelectric materials with high value of $Z T$.

Recently, great progress has been achieved in improving the thermoelectric 
properties of nanostructural organic materials [13] [14] [15]. It can be proved to enhance the thermoelectric properties whether using nanofibers and nanotubes as fillers or coating the nano-structured inorganic particles on the polymer layer. Christian, et al., prepared P3HT/MW-CNT composites by casting method and then estimated the maximal $P F$ [13]. When a filler content of CMW-CNT was 8 wt\%, the maximum value of $P F$ was $6 \pm 2 \mu \mathrm{Wm}^{-1} \mathrm{~K}^{-2}$. Instead, when a filer content of CSW-CNT was from $42 \mathrm{wt} \%$ to $81 \mathrm{wt} \%$, the maximum value of $P F$ was 95 $\pm 12 \mu \mathrm{Wm}^{-1} \mathrm{~K}^{-2}$. Cai, et al., prepared PEDOT and PbTe-modified PEDOT nanotubes, using an in situ interfacial polymerization method [14]. Their pure PEDOT was an n-type thermoelectric material, with larger value of Seebeck coefficient $\left(-4088 \mu \mathrm{VK}^{-1}\right)$ and lower electrical conductivity $\left(0.064 \mathrm{Sm}^{-1}\right)$.

PEDOT is believed to be one of the most promising TE materials among the conducting polymers [1] [16] [17] [18] [19] [20]. The experiments indicated that some factors affect the thermoelectric properties of 2D films [5] [7] [9] [21] [22] [23] [24]. For example, physical doping can adjust the concentration of carriers. Controlling of impurities and defects in the material can effectively regulate the carrier mobility. The organic solvents can be used to reduce the barriers of the electron transport. The electrical conductivity of PEDOT: PSS films was enhanced after post-processing using the organic solvent [25] [26] [27], such as dimethyl sulfoxide (DMSO) and methanol. The structure of PEDOT chain changed from random coil to a linear due to the removal of excess non-ionized PSS, leading to a great improvement of the electrical conductivity. After post-processing, The electrical conductivity of PEDOT: PSS films may increase by about 3 orders of magnitude [25], the Seebeck coefficient could change in varying degrees [5] [23] [24]. Seebeck coefficient and electrical conductivity of PEDOT: PSS or PEDOT-Tos film can be usually affected by the treatment of dopant, carrier concentration and mobility. The organic thermoelectric material with high $P F$ may be obtained through controllable doping and oxidation level [5] [7] [21] [22] [23] [24] [28] [29] [30].

Glass fiber is commonly used as a substrate due to its high thermal resistant, electrical insulation, anti-chemical corrosion and other excellent performance [31]. The research on glass fiber in the literature mainly focused on the preparation of conductive glass fiber, the electrical conductivity, the magnetic properties, and the mechanical properties of the materials [32] [33] [34]. A. J. Attias, et al., coated polypyrrole on the surface of glass fibers pre-treated with a coupling agent, and the conductivity of materials was $150-200 \mathrm{Scm}^{-1}$ [35]. Guo, et al., deposited $\mathrm{Ni}$ on glass fibers by electroless plating, then glass fibers coated with polypropylene/Ni were prepared by solution mixing [36]. The electrical conductivity of polypropylene/Ni/glass fiber was $8.7 \mathrm{Scm}^{-1}$. In recent years, researchers have successfully synthesized flexible inorganic nanocrystal-coated glass fibers using a scalable solution-phase deposition method to coat PbTe nanocrystals onto the surface of glass fibers [2]. It was regrettable that the electrical conductivity $\left(104 \mathrm{Sm}^{-1}\right)$ was lower than that of the same component. However, few re- 
searchers try to apply glass fibers to organic thermoelectric materials. In addition, the fiber-like one-dimensional TE material may be applied in the area of waste heat recovery owning to their roll-to-roll processability. In order to improve the thermoelectric efficiency of thermoelectric devices, exploring low-cost, high-performance organic thermoelectric materials is very necessary and significant.

In this work, poly(3,4-ethylenedioxythiophene) was coated on the surface of glass fiber with in situ polymerization. We have prepared 1D flexible composite thermoelectric materials based on PEDOT: p-TSA /glass fiber and hope to bring out the performance of the "electron conductor, photon glass". The synthesis conditions are investigated and the material was further treated with methanol. The electric conductivity and Seebeck coefficient are measured.

\section{Experimental Procedure}

\subsection{Materials}

All chemicals as following, and just 3,4-ethylenedioxythiophene was used with further purification. 3,4-ethylenedioxythiophene (EDOT, $>98 \%$, Beijing Coupling Technology Co., Ltd., China), p-toluenesulfonic acid (p-TSA, AR, Tianjin Fuchen Chemical Reagent Factory, China), ammonium persulfate (APS, $\geq 99 \%$, Beijing Kehua Jingwei Technology Co., Ltd, China), methanol (MeOH, AR, Beijing Coupling Technology Co., Ltd., China). Glass fiber used a bundle with 1000 monofilaments which length are $3 \mathrm{~cm}$ and the diameter are $10 \mu \mathrm{m}$.

\subsection{Preparation PEDOT: p-TSA/Glass Fiber}

Preparation the 1D organic thermoelectric materials of PEDOT: p-TSA/glass fiber, which involves the following procedures: 1) EDOT was distilled under vacuum $\left(0.095 \mathrm{KPa}, 102^{\circ} \mathrm{C}\right)$ and the purity of EDOT reached to $99.9 \%$. 2) Preparation of APS solution with different concentrations, 5, 7, 9, 11, 13, $15 \mathrm{mmol} \cdot \mathrm{L}^{-1}$. (mM), respectively. 3) EDOT and p-TSA solution were mixed together and then stirred for $20 \mathrm{~min}$. Glass fibers fabricated into a bundle of $3 \mathrm{~cm}$ long were placed in EDOT: p-TSA mixture. 4) The APS solution was added slowly dropwise to the dispersion under magnetic stirring at $\sim 15^{\circ} \mathrm{C}$. 5) After stirring for 9.5 hours, the material was washed several times by the distilled water, then drying under vacuum at $60^{\circ} \mathrm{C}$ for $24 \mathrm{~h}$. 6) For post-processing, the pristine sample was dried for 20 minutes at $130^{\circ} \mathrm{C}$ in the oven, then immersed in methanol solution for 10 minutes and dried for $5 \mathrm{~h}$ at $130^{\circ} \mathrm{C}$ in the vacuum oven. 7) The samples were measured thermoelectric properties at room temperature. We provide the two kinds of samples with better thermoelectric properties than that of the other samples which are measured in this work. The two kinds of samples that are measured are defined P-34 and P-76, in which the mass fraction of p-TSA relative to $\mathrm{EDOT}$ is $34 \%$ and $76 \%$ in the reaction, or the molar ratios of $\mathrm{p}-\mathrm{TSA}$ and EDOT is 60:1 and 130:1, respectively. According to the concentration of APS from 5 to $15 \mathrm{mM}$, the P-34 sample are marked as Sample A1-A6 in turn. 
Figure 1 is schematic diagram of the formation process of $1 \mathrm{D}$ thermoelectric materials PEDOT: p-TSA/glass fiber. The cylindrical represents monofilament fiber, the black dots in "Preliminary polymerization" mean oligomers of EDOT.

\subsection{Characterization}

FT-IR (Bruker Vector 22) spectrum of PEDOT: p-TSA (Figure 5) is used to characterize the polymer chain group type of the samples. The morphology of the samples is characterized by scanning electron microscopy (S-4700). The electrical conductivity of monofilament fiber is measured with a Keithley2000 Multimeter (Keithley Instruments Inc., USA) using a standard four-probe method at room temperature. The electrical conductivity of monofilament fiber is used to represent that of the bundle. The preparation of the measured sample is as following. The monofilament fiber is fixed on the glass and connected with four copper wires using silver paint. After that, it is heated for $80 \mathrm{~min}$ at $110^{\circ} \mathrm{C}$. The Seebeck coefficient of the sample is measured on the apparatus (Keithley2400) [37]. In order to prepare the tested sample, the fibers are fabricated into a bundle including about 1000 monofilaments which length are $3 \mathrm{~cm}$ firstly. Two thermocouples are stuck on the bundle using silver paint and then heated for 80 $\min$ at $110^{\circ} \mathrm{C}$ to fix the thermocouples firmly. Eventually the sample is laid on measurement substrate after cooling, and fixed it by sandwiching as shown in Figure 2. The voltage difference and temperature difference are detected between

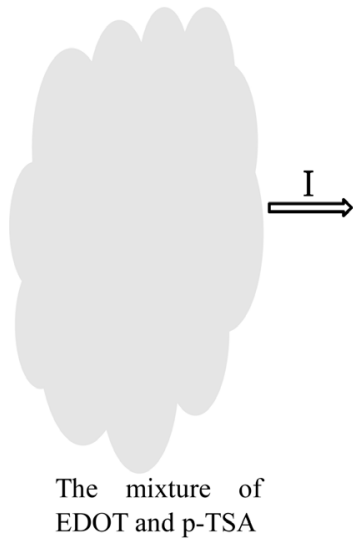

I : Addition of glass fibers

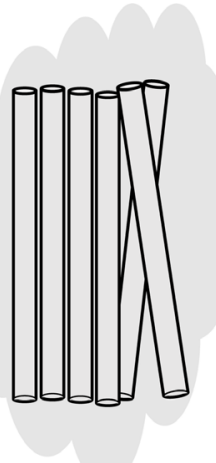

Glass fibers + EDOT: $p$-TSA

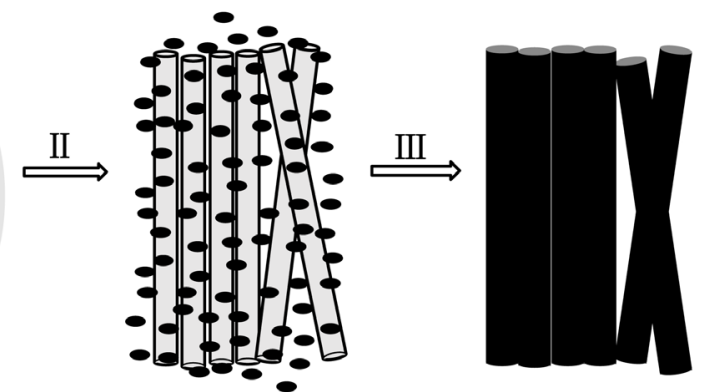

Preliminary polymerization PEDOT: $\mathrm{p}$-TSA/glass fibers

II : Addition of APS solution

III: Polymerization for $9.5 \mathrm{~h}$

Figure 1. The schematic diagram of the formation process of PEDOT: p-TSA/glass fiber.

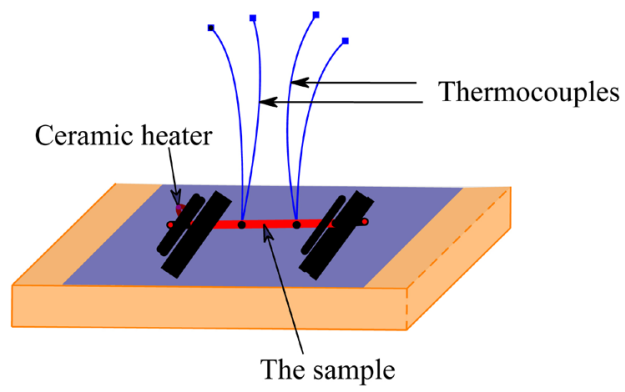

Figure 2. The diagrammatic drawing of fixing on sample (PEDOT: p-TSA/glass fiber) for measuring $S$. 
the hot and the cold side. To ensure the accuracy of the measurement, each sample has been measured for 3 times.

\subsection{Preparation PEDOT: p-TSA/Glass Fiber}

FT-IR (Bruker Vector 22) spectrum of PEDOT: p-TSA (Figure 5) is used to characterize the polymer chain group type of the samples. The morphology of the samples is characterized by scanning electron microscopy (S-4700). The electrical conductivity of monofilament fiber is measured with a Keithley 2000 Multimeter (Keithley Instruments Inc., USA) using a standard four-probe method at room temperature. The electrical conductivity of monofilament fiber is used to represent that of the bundle. The preparation of the measured sample is as following. The monofilament fiber is fixed on the glass and connected with four copper wires using silver paint. After that, it is heated for $80 \mathrm{~min}$ at $110^{\circ} \mathrm{C}$. The Seebeck coefficient of the sample is measured on the apparatus (Keithley 2400) [37]. In order to prepare the tested sample, the fibers are fabricated into a bundle including about 1000 monofilaments which length are $3 \mathrm{~cm}$ firstly. Two thermocouples are stuck on the bundle using silver paint and then heated for 80 min at $110^{\circ} \mathrm{C}$ to fix the thermocouples firmly. Eventually the sample is laid on measurement substrate after cooling, and fixed it by sandwiching as shown in Figure 2. The voltage difference and temperature difference are detected between the hot and the cold side. To ensure the accuracy of the measurement, each sample has been measured for 3 times.

\section{Results and Discussion}

\subsection{Image of PEDOT: p-TSA/Glass Fiber}

Figure 3(a) shows high magnification microscope image of the monofilament glass fiber and PEDOT: p-TSA/glass fiber. The bottom of the picture is the PEDOT: p-TSA/glass fiber which darkness represents the PEDOT: p-TSA coating layer on the glass fiber. The above is the raw material glass fiber that is light color. In Figure 3(b), it is the sample to be measured for S. PEDOT: p-TSA/glass fiber material that we have prepared is flexible (Figure 3(c)).

\subsection{FT-IR Analysis}

The powder sample of pristine PEDOT: p-TSA is obtained according to the method of solution polymerization. In the FT-IR spectrum of PEDOT: p-TSA (see Figure 4, black line), the asymmetric $\mathrm{C}=\mathrm{C}$ stretching peak could be observed at $1517.4 \mathrm{~cm}^{-1}$. C-O-C deformation peak and oxyethylene ring deformation stretching peak are shown at $1052.7 \mathrm{~cm}^{-1}$ and $851 \mathrm{~cm}^{-1}$, respectively [17]. The peak of C-S-C deformation of PEDOT is observed at 981.5 and $839.1 \mathrm{~cm}^{-1}$ in the FT-IR spectrum. The absorption peak and stretching vibrational absorption peak of methyl are at $1399.1 \mathrm{~cm}^{-1}$ and $2800 \mathrm{~cm}^{-1}$, respectively. And some signal peaks may be weakened. The absorption peak and stretching vibrational absorption peak of sulfonic acid group are at $686 \mathrm{~cm}^{-1}$ and $1198.9 \mathrm{~cm}^{-1}$, respectively. The 

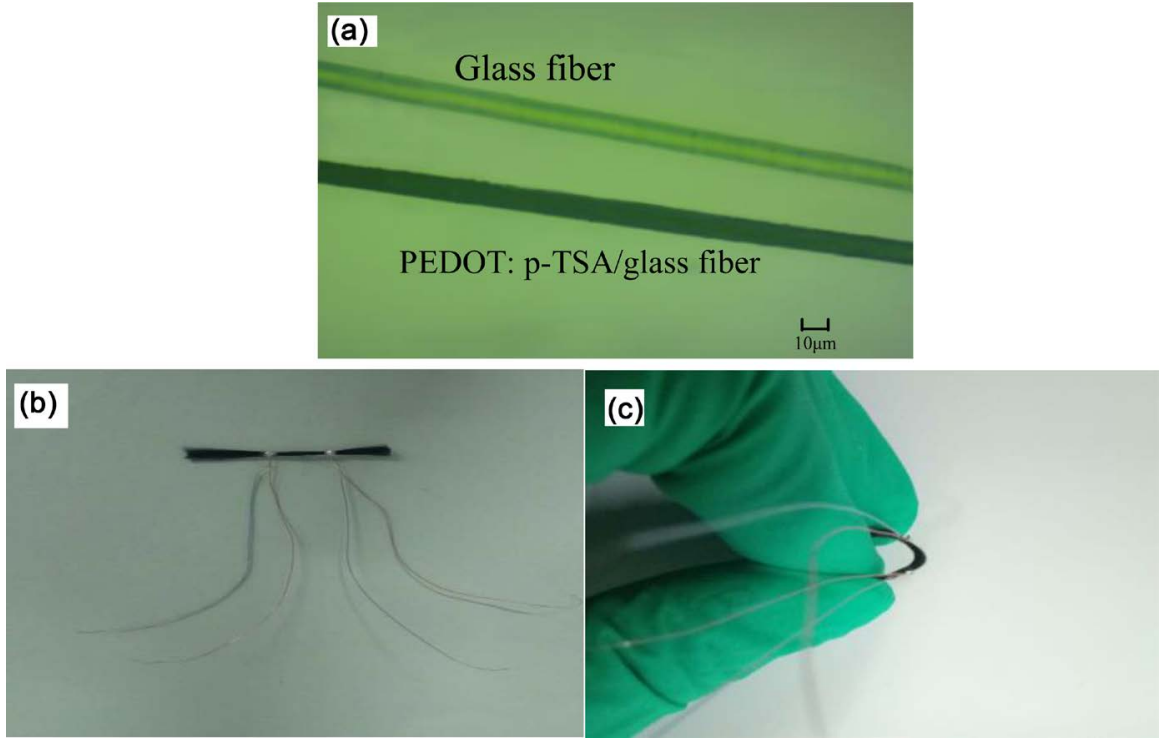

Figure 3. (a) High magnification microscope image of the glass fiber and PEDOT: p-TSA/glass fiber. (b) (c) Digital image of a bunch of PEDOT: p-TSA/glass fiber with two thermocouples.

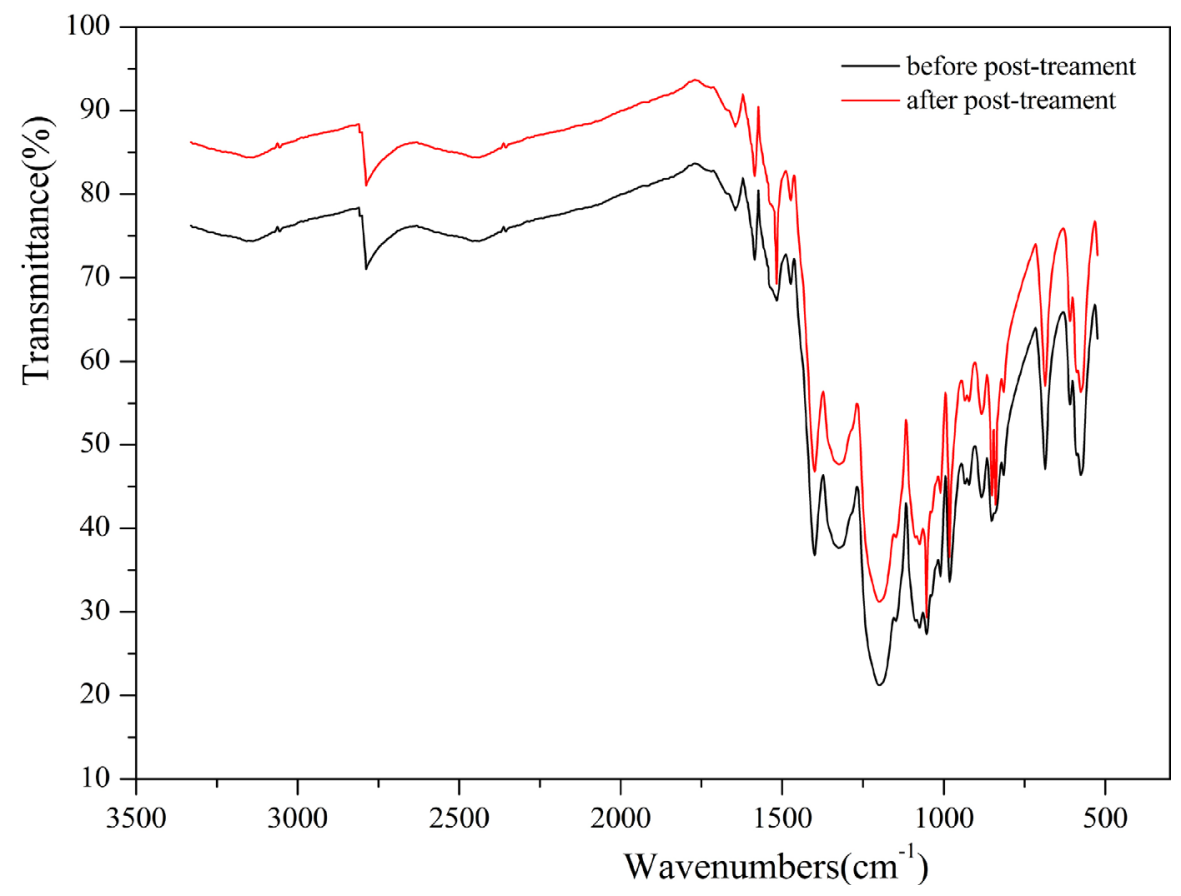

Figure 4. FT-IR spectrum of PEDOT: p-TSA.

vibrational absorption peak of the benzene ring is at $1645 \mathrm{~cm}^{-1}, 1473.6 \mathrm{~cm}^{-1}$ and $1580 \mathrm{~cm}^{-1}$. In our opinion, p-TSA is doped in PEDOT. In the FT-IR spectrum of the sample after post-treatment (see Figure 4, red line), absorption peak of the group doesn't change, only the absorption intensity of methyl, benzene ring and sulfonic acid group decrease.

Referring to the PEDOT-Tos film [7], the chemical structure of PEDOT: p-TSA in PEDOT: p-TSA/glass fiber material was shown in Figure 5. 


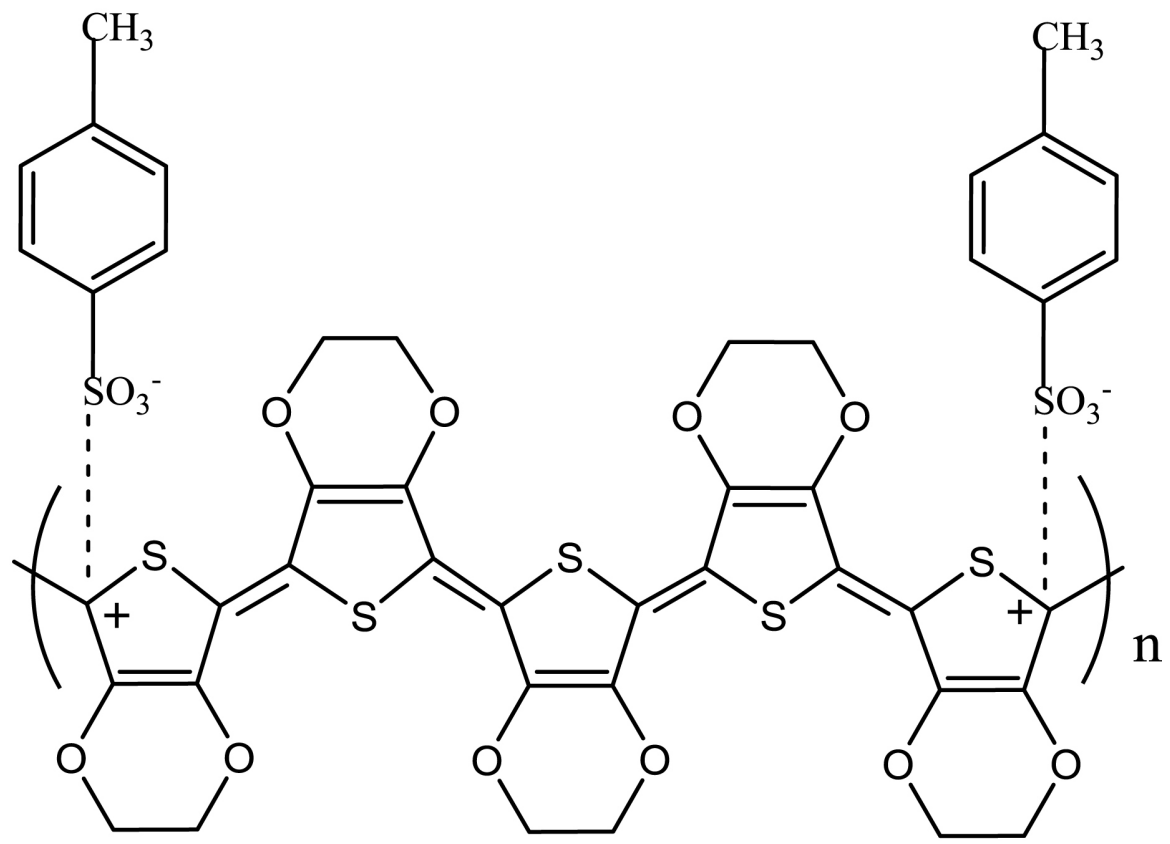

Figure 5. Chemical structure of PEDOT: p-TSA.

\subsection{Scanning Electron Microscopic Analysis}

The SEM image (Figure 6) shows that the surface of organic coating appears to be considerable roughness and continuous for all samples which are after post-treatment. The morphology for all samples that are oxidized with the same concentration of APS is generally similar with the view of SEM images. The SEM images of P-34 is shown in Figure 6. When the concentration of APS is $7 \mathrm{mM}$ (Sample A2), $9 \mathrm{mM}$ (Sample A3), and $11 \mathrm{mM}$ (Sample A4), the image is shown in Figures 6(a)-(c), respectively. With the increase of the concentration of oxidants, we observe different morphology. Therefore, the difference in oxidant concentration leads to different morphology of the sample. It is flower cluster in Figure 6(a), Figure 6(b) and like-scaly in Figure 6(c). The morphology of Sample A1 $(5 \mathrm{mM})$ is similar to that of Sample A2, while the size of particles is smaller than that of Sample A2. The morphology of Sample A5 (13 mM) and Sample A6 (15 mM) are similar to that of Sample A4, but the size of particles is bigger than that of Sample A4. The higher concentration of the oxidant could make the polymerization reactions occur more quickly, which results in the shorter polymer chains formed and it may be intertwining each other, so the large particles were formed. While the oxidant concentration is lower, polymerization occurs more slowly, which results in the longer polymer chains formed, and the small particles formed. The polymer of PEDOT: p-TSA is stacked in a layer. The coating is fabricated with the particles of the polymer that are agglomerated on the glass fiber. The particles are formed from the self-curly polymer chains. There is existence of van der Waals force between the particles. It is quite clear that the interface exists among these particles (see Figures 6(a)-(c)). The sample with the best thermoelectric property was measured to obtain the thickness 


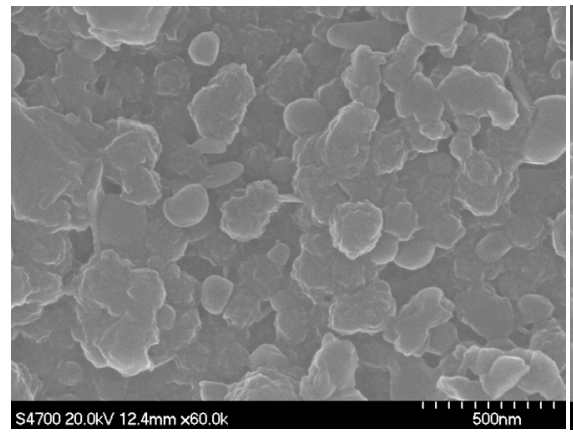

(a)

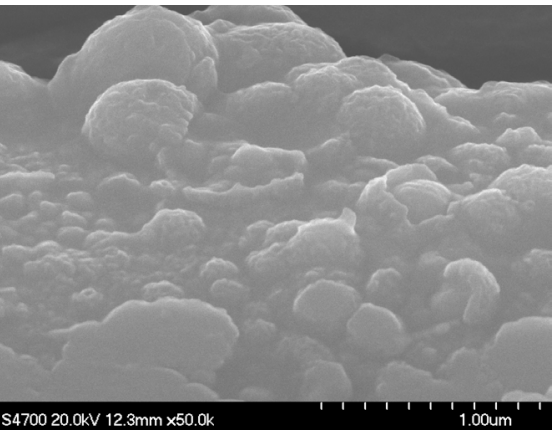

(b)

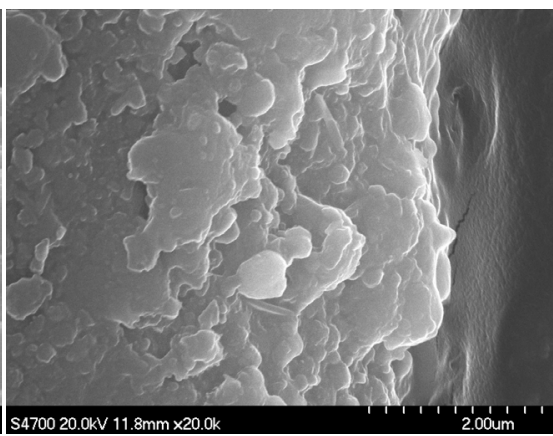

(c)

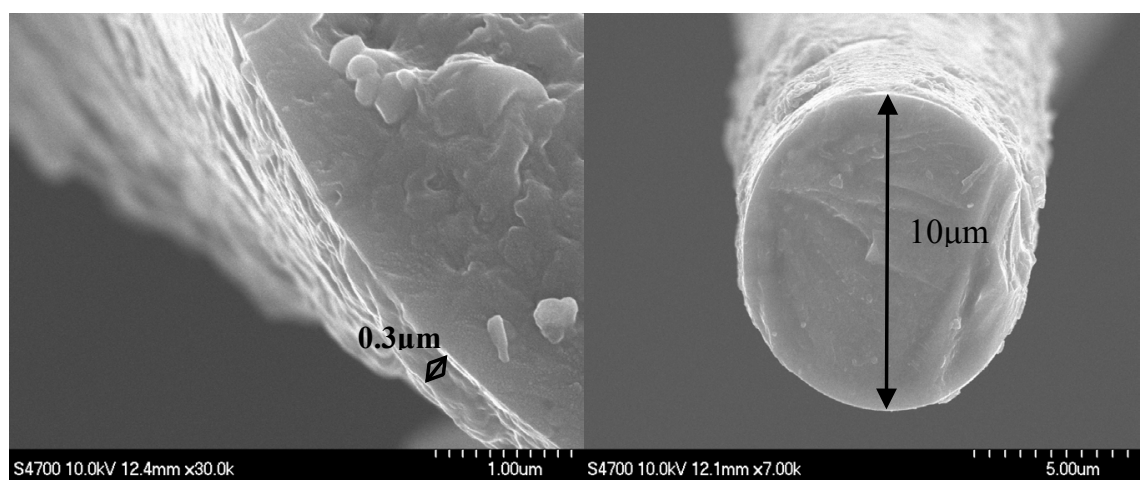

(d)

(e)

Figure 6. (a)-(c) SEM image of PEDOT: p-TSA/glass fiber sample A2, sample A3 and sample A4, respectively. (d) (e) SEM image of PEDOT: p-TSA/glass fiber. The diameter of fiber is about $10 \mu \mathrm{m}$ and the thickness of coating is about $0.3 \mu \mathrm{m}$.

of organic coating. SEM images of Sample A4 show that the diameter of glass fiber is $10 \mu \mathrm{m}$ (Figure 6(d)) uniformly, and the thickness of organic coating is approximately $0.3 \mu \mathrm{m}$ (Figure $6(\mathrm{e})$ ).

\subsection{Characterization of Thermoelectric Properties}

At Figure 7, the lines with full symbol and the lines with empty symbol are used to present thermoelectric properties of the samples of P-34 and P-76, respectively. We explore an effect of the concentration of oxidant (APS) from 5 to $15 \mathrm{mM}$ on thermoelectric properties. As for P-34, when the concentration of APS increases from 5 to $11 \mathrm{mM}$, the electric conductivity (Figure 7(a)) of the PEDOT: p-TSA/glass fiber increases from $21 \mathrm{Sm}^{-1}$ to $169 \mathrm{Sm}^{-1}$, then decreases to $91 \mathrm{Sm}^{-1}$ with the concentration of APS increasing to $15 \mathrm{mM}$. However, the Seebeck coefficient (Figure 7(a)) shows a decreasing trend from $32 \mu \mathrm{VK}^{-1}$ to $19 \mu \mathrm{VK}^{-1}$, with the concentration of APS increasing from 5 to $11 \mathrm{mM}$. Then the Seebeck coefficient increases to $22 \mu \mathrm{VK}^{-1}$ with the concentration of APS increasing to $15 \mathrm{mM}$. As for P-76, the concentration of APS increases from 5 to $9 \mathrm{mM}$, the electric conductivity (Figure 7(a)) of the PEDOT: p-TSA/glass fiber increases from 20 to $133 \mathrm{Sm}^{-1}$. Then it decreases to $84 \mathrm{Sm}^{-1}$ with the concentration of APS increasing to $15 \mathrm{mM}$. The Seebeck coefficient (Figure 7(a)) illustrates an opposite trend to that shown in Figure 7(a). As the concentration of APS increases from 5 to 9 $\mathrm{mM}$, the Seebeck coefficient (Figure 7(a)) shows an increasing trend from 25 to 


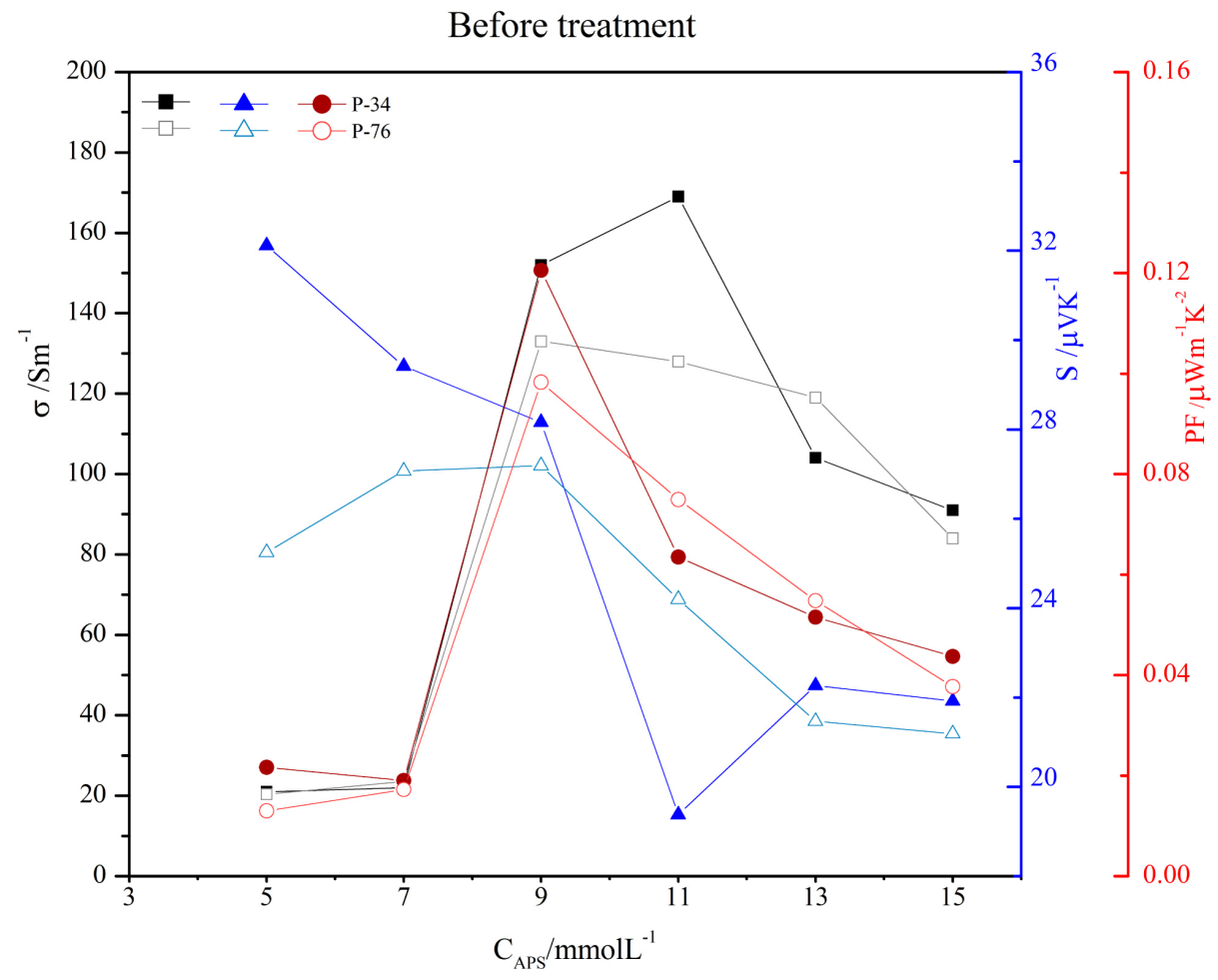

(a)

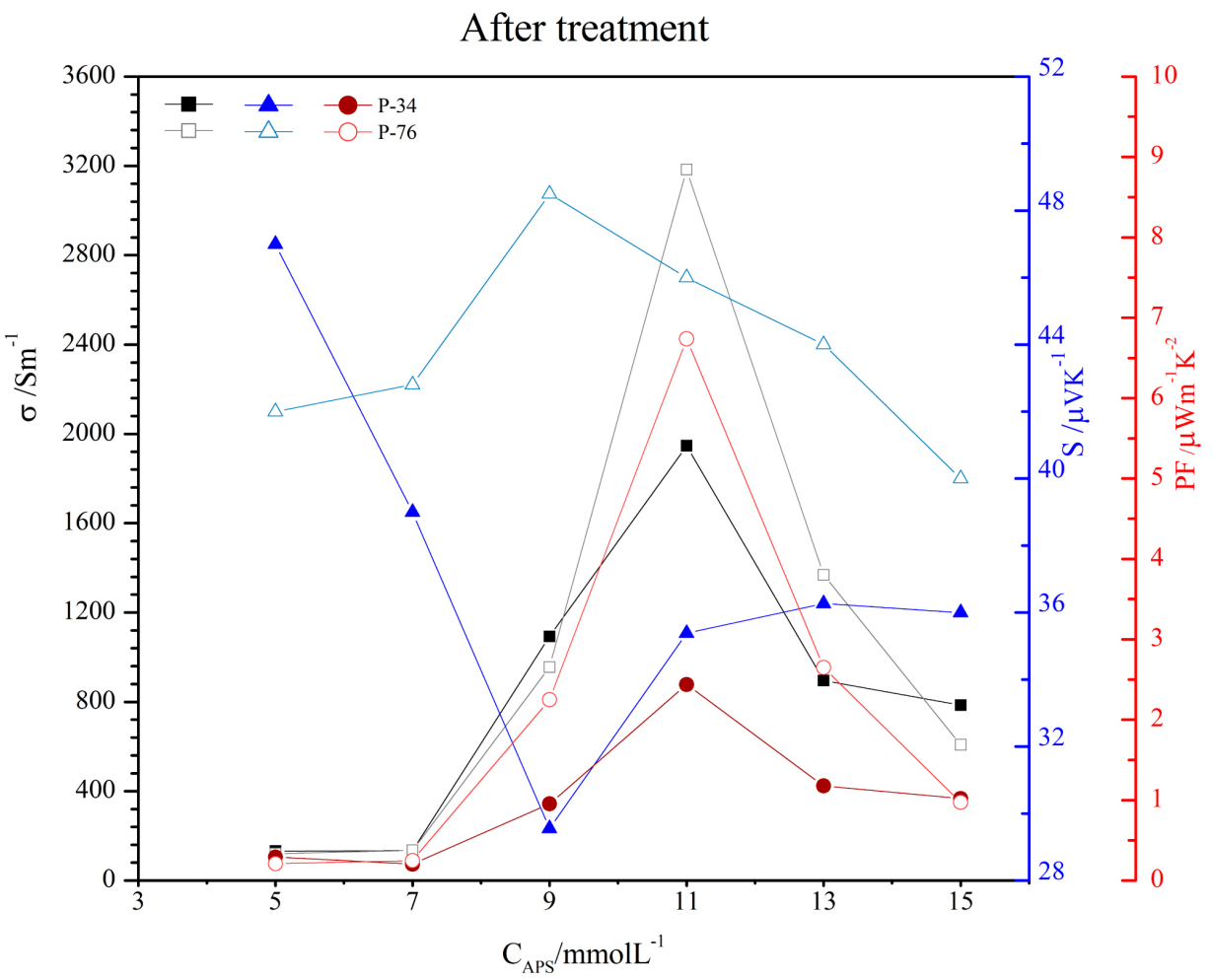

(b)

Figure 7. Thermoelectric properties of (PEDOT: p-TSA)/glass fiber of different samples (a) before and (b) after treatment by methanol. Full symbol for P-34 sample, empty symbol for P-76 sample. 
27.3 $\mu \mathrm{VK}^{-1}$. After that, it decreases to $21 \mu \mathrm{VK}^{-1}$ with the concentration of APS increasing to $15 \mathrm{mM}$. When the concentration of oxidant is the same, the maximum $P F$ of sample P-34 $\left(0.12 \mu \mathrm{Wm}^{-1} \mathrm{~K}^{-2}\right)$ with low doping mass fraction are generally higher than that of sample P-76 $\left(0.10 \mu \mathrm{Wm}^{-1} \mathrm{~K}^{-2}\right)$. The maximum electric conductivity is $169 \mathrm{Sm}^{-1}$ when the concentration of APS is $11 \mathrm{mM}$. And the maximum Seebeck coefficient is $32 \mu \mathrm{VK}^{-1}$ when the concentration of APS is $5 \mathrm{mM}$. The maximum power factor $\left(0.12 \mu \mathrm{Wm}^{-1} \mathrm{~K}^{-2}\right)$ is shown in Figure 7 (a) with $9 \mathrm{mM}$ APS. It illustrates that the concentration of oxidant affects the microstructure of the polymer, also its thermoelectric properties.

After post-processing, the Seebeck coefficient and electrical conductivity have been enhanced. For the sample P-34, as the concentration of APS increases from 5 to $11 \mathrm{mM}$, the electric conductivity (Figure 7(b)) increases from 132 to 1947 $\mathrm{Sm}^{-1}$. Then it decreases to $785 \mathrm{Sm}^{-1}$ with the concentration of APS increasing to $15 \mathrm{mM}$. However, the Seebeck coefficient (Figure 7(b)) shows a decreasing trend from 47 to $30 \mu \mathrm{VK}^{-1}$, with the concentration of APS increasing from 5 to 9 $\mathrm{mM}$. Next it increases to $36 \mu \mathrm{VK}^{-1}$ with the concentration of APS increasing to $15 \mathrm{mM}$. For the sample P-76, as the concentration of APS increases from 5 to 11 $\mathrm{mM}$, the electric conductivity (Figure $7(\mathrm{~b})$ ) of the PEDOT: p-TSA/glass fiber increases from 120 to $3184 \mathrm{Sm}^{-1}$. Then it drops to $608 \mathrm{Sm}^{-1}$ with the concentration of APS increasing to $15 \mathrm{mM}$. As the concentration of APS increases from 5 to $9 \mathrm{mM}$, the Seebeck coefficient (Figure 7(b)) shows an increasing trend from 42 to $48.5 \mu \mathrm{VK}^{-1}$. After that, it decreases to $40 \mu \mathrm{VK}^{-1}$ with the concentration of APS increasing to $15 \mathrm{mM}$. We have obtained the optimal value of thermoelectric performance of the sample that the mass fraction of p-TSA in reagent is $76 \%$. The maximum electric conductivity is $3184 \mathrm{Sm}^{-1}$ corresponding the $P F$ is 6.74 $\mu \mathrm{Wm}^{-1} \mathrm{~K}^{-2}$ (Figure $7(\mathrm{~b})$ ) when the concentration of APS is $11 \mathrm{mM}$. And the maximum Seebeck coefficient is $48.5 \mu \mathrm{VK}^{-1}$ at $9 \mathrm{mM}$ APS. After post-processing, the maximum $P F\left(6.74 \mu \mathrm{Wm}^{-1} \mathrm{~K}^{-2}\right)$ is about 56 times larger than that of the pristine sample $\left(0.12 \mu \mathrm{Wm}^{-1} \mathrm{~K}^{-2}\right)$.

This work attempts to prepare the 1D TE organic-inorganic composites materials employing the conductivity of surface layer PEDOT and the adiabaticity of core glass fiber. A possible explain for above results is suggested. Before post treatment, it is obtained the higher value of $P F\left(0.12 \mu \mathrm{Wm}^{-1} \mathrm{~K}^{-2}\right)$ in which the concentration of APS is $9 \mathrm{mM}$. We speculated the reasons as following. The higher concentration of the oxidant could make the more polymerization reactions occur, which results in the shorter polymer chains formed and it may be intertwining each other. While the oxidant concentration is lower, the polymer chain may be longer and self-winding. Only the oxidant concentration is an appropriate value, the number of polymer chains with suitable length are formed and beneficial to the better the transmission of the charge carriers. Thus the concentration of oxidizing agent has a good value for heightening thermoelectric properties.

Methanol is used for post-treatment to remove the excess p-TSA molecules 
that are not ionized as well as make the PEDOT chain more extended-coil or linear [25] and increase the delocalization of $\pi$-electron in polymer chains [27], which is beneficial to the transmission of electrons. Regrettably, we couldn't observe directly the obvious change in the degree of chain structural order, but it was resulted that post treatment brought out the ordered connected PEDOT chain [25]. The highest value of electrical conductivity $\left(3184 \mathrm{Sm}^{-1}\right.$, see Figure 7 (b)) of our materials is lower than the value of PEDOT-Tos film $\left(30,000 \mathrm{Sm}^{-1}\right)$ in the reference [7], due to the PEDOT: p-TSA coating layer is only $0.3 \mu \mathrm{m}$ thick and the glass fiber is insulative. Organic conductive layer just accounted for a relatively small proportion of the composites materials. If increasing the thickness of the conductive layer, the electrical conductivity of the materials will be increased. PEDOT: p-TSA/glass fiber shows an enhanced Seebeck coefficient which is about 1.5 times larger than the PEDOT: PSS films $\left(\sim 20 \mu \mathrm{VK}^{-1}\right)$ [21] [23] [24] and is comparable to Seebeck coefficient of the PEDOT: PSS nanofilm and pristine PEDOT:Tos film [7] [22] at room temperature. After post processing, the maximum value of $\mathrm{S}$ goes up from 32 to $48.5 \mu \mathrm{VK}^{-1}$ and the maximum value of $P F$ goes up from 0.12 to $6.74 \mu \mathrm{Wm}^{-1} \mathrm{~K}^{-2}$ in our work. This could be explained as that post-processing results in sequential conformational changes of polymer chains. In proximity of the Fermi level, $S$ increases as the steeper distribution of the density of state (DOS) of electron [38] [39] [40] [41].

The schematic diagram of carries transport of PEDOT: p-TSA/glass fiber material was shown in the Figure 8 referring to the SEM image of material. Energy filtering from the interface of particles may change the Seebeck coefficient and at the same time phonons could scatter to decrease thermal conductivity. As for the thermal conductivity $\kappa$, Liu Y et al. [42] reviewed a number of methods for testing one-dimensional nanostructural materials. It is difficult to test the thermal conductivity $\kappa$ of the fiber-like TE materials at present. The further work is underway.

\section{Conclusion}

PEDOT: p-TSA/glass fiber, one-dimensional organic-inorganic composite thermoelectric materials, was prepared by coating the 3,4-ethylenedioxythiophene on the surface of glass fiber using in situ polymerization method. Excessively high or low mass fractions (p-TSA relative to EDOT) can affect carrier transport and reduce thermoelectric performance, when removing excess dopants will increase

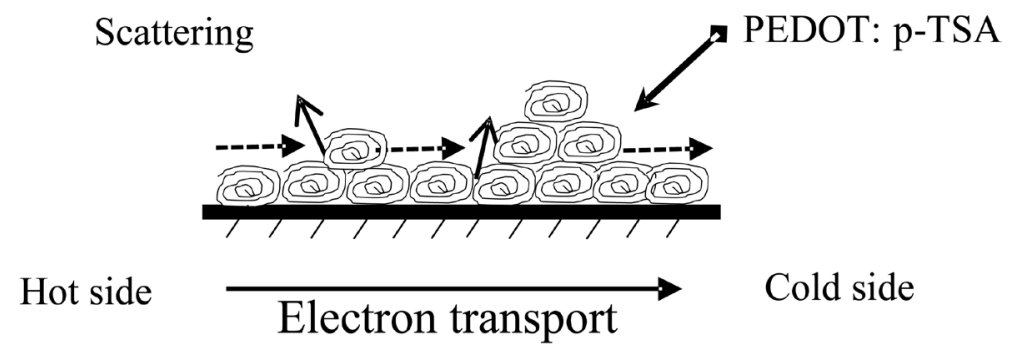

Figure 8. Schematic diagram of carries transport of PEDOT: p-TSA/glass fiber material. 
$P F$ in this work. The effects of oxidant concentration (APS) with an appropriate value on thermoelectric properties are that the number of polymer chains with suitable length is formed and beneficial to the better transmission of the charge carriers, which improve the $P F$ finally. After post-processing using methanol solution, the maximal $S$ increases from $32 \mu \mathrm{VK}^{-1}$ to $48.5 \mu \mathrm{VK}^{-1}$, and the maximal $\sigma$ increases from $169 \mathrm{Sm}^{-1}$ to $3184 \mathrm{Sm}^{-1}$. The optimized $P F$ was $6.74 \mu \mathrm{Wm}^{-1} \mathrm{~K}^{-2}$. We verified that post-processing enhanced the TE properties of materials.

\section{References}

[1] Chen, G., Xu, W. and Zhu, D. (2017) Recent Advances in Organic Polymer Thermoelectric Composites. Journal of Materials Chemistry C, 5, 4350-4360. https://doi.org/10.1039/C6TC05488A

[2] Liang, D., Yang, H., Finefrock, S.W. and Wu, Y. (2012) Flexible Nanocrystal-Coated Glass Fibers for High-Performance Thermoelectric Energy Harvesting. Nano Letters, 12, 2140-2145. https://doi.org/10.1021/nl300524j

[3] Li, C., Jiang, F., Liu, C., Wang, W., Li, X., Wang, T. and Xu, J. (2017) A Simple Thermoelectric Device Based on Inorganic/Organic Composite Thin Film for Energy Harvesting. Chemical Engineering Journal, 320, 201-210. https://doi.org/10.1016/j.cej.2017.03.023

[4] Dresselhaus, M.S., Chen, G., Tang, M.Y., Yang, R., Lee, H., Wang, D., Ren, Z., Fleurial, J.P. and GognaNew, P. (2007) Directions for Low-Dimensional Thermoelectric Materials. Advance Materials, 19, 1043-1053. https://doi.org/10.1002/adma.200600527

[5] Kim, G.H., Shao, L., Zhang, K. and Pipe, K.P. (2013) Engineered Doping of Organic Semiconductors for Enhanced Thermoelectric Efficiency. Nature Materials, 12, 719-723. https://doi.org/10.1002/adma.200600527

[6] Sun, Y., Qiu, L., Tang, L., Geng, H., Wang, H., Zhang, F., Huang, D., Xu, W., Yue, P., Guan, Y., et al. (2016) Flexible n-Type High-Performance Thermoelectric Thin Films of Poly(nickel-ethylenetetrathiolate) Prepared by an Electrochemical Method. Advance Materials, 28, 3351-3358. https://doi.org/10.1002/adma.201505922

[7] Bubnova, O., Khan, Z.U., Malti, A., Braun, S., Fahlman, M., Berggren, M. and Crispin, X. (2011) Optimization of the Thermoelectric Figure of Merit in the Conducting Polymer Poly(3,4-ethylenedioxythiophene). Nature Materials, 10, 429-433. https://doi.org/10.1038/nmat3012

[8] Si, H., Wang, Y., Yan, Y. and Zhang, G. (2012) Structural, Electronic, and Thermoelectric Properties of InSe Nanotubes: First-Principles Calculations. The Journal of Physical Chemistry C, 116, 3956-3961. https://doi.org/10.1021/jp210583f

[9] Xi, J., Zhao, T., Wang, D. and Shuai, Z. (2014) Tunable Electronic Properties of Two-Dimensional Transition Metal Dichalcogenide Alloys: A First-Principles Prediction. The Journal of Physical Chemistry Letters, 5, 285-291. https://doi.org/10.1021/jz402375s

[10] Wang, Y., Zhou, J. and Yang, R. (2011) Thermoelectric Properties of Molecular Nanowires. The Journal of Physical Chemistry C, 115, 24418-24428. https://doi.org/10.1021/jp208490q

[11] Vukmirovi, N. and Wang, L. (2010) Carrier Hopping in Disordered Semiconducting Polymers: How Accurate Is the Miller-Abrahams Model? Applied Physics Letters, 97, Article ID: 043305. https://doi.org/10.1063/1.3474618 
[12] Shi, W., Shuai, Z. and Wang, D. (2017) Tuning Thermal Transport in Chain-Oriented Conducting Polymers for Enhanced Thermoelectric Efficiency: A Computational Study. Advanced Functional Materials, 27, 1702847. https://doi.org/10.1002/adfm.201702847

[13] Bounioux, C., Díaz-Chao, P., Campoy-Quiles, M., Martín-González, M.S., Goñi, A.R., Yerushalmi-Rozen, R. and Müller, C. (2013) Thermoelectric Composites of Poly(3-hexylthiophene) and Carbon Nanotubes with a Large Power Factor. Energy \& Environmental Science, 6, 918-925. https://doi.org/10.1039/c2ee23406h

[14] Wang, Y., Cai, K. and Yao, X. (2011) Facile Fabrication and Thermoelectric Properties of PbTe-Modified Poly(3,4-ethylenedioxythiophene) Nanotubes. ACS Applied Materials Interfaces, 3, 1163-1166. https://doi.org/10.1021/am101287w

[15] Kim, D., Kim, Y., Choi, K., Grunlan, J.C. and Yu, C. (2010) Improved Thermoelectric Behavior of Nanotube-Filled Polymer Composites with Poly(3,4-ethylenedioxythiophene) Poly(styrenesulfonate). ACS Nano, 4, 513-523.

https://doi.org/10.1021/nn9013577

[16] Zhang, Q., Sun, Y., Xu, W. and Zhu, D. (2014) Organic Thermoelectric Materials: Emerging Green Energy Materials Converting Heat to Electricity Directly and Efficiently. Advanced Materials, 26, 6829-6851.

https://doi.org/10.1002/adma.201305371

[17] Yu, X., Su, X., Yan, K., Hu, H., Peng, M., Cai, X. and Zou, D. (2016) Stretchable, Conductive, and Stable PEDOT-Modified Textiles through a Novel in Situ Polymerization Process for Stretchable Supercapacitors. Advanced Materials Technologies, $1,1-8$.

[18] Xu, H., Pang, X., He, Y., He, M., Jung, J., Xia, H. and Lin, Z. (2015) An Unconventional Route to Monodisperse and Intimately Contacted Semiconducting Organic-Inorganic Nanocomposites. Angewandte Chemie, 54, 4636-4640. https://doi.org/10.1002/anie.201500763

[19] Rice, A.H., Giridharagopal, R., Zheng, S.X., Ohuchi, F.S., Ginger, D.S. and Luscombe, C.K. (2011) Controlling Vertical Morphology within the Active Layer of Organic Photovoltaics Using Poly(3-hexylthiophene) Nanowires and Phenyl-C61-butyric Acid Methyl Ester. ACS Nano, 5, 3132-3140. https://doi.org/10.1021/nn2002695

[20] Bharti, M., Singh, A., Samanta, S. and Aswal, D.K. (2018) Conductive Polymers for Thermoelectric Power Generation. Progress in Materials Science, 93, 270-310. https://doi.org/10.1016/j.pmatsci.2017.09.004

[21] Tsai, T.C., Chang, H.C., Chen, C.H., Huang, Y.C. and Whang, W.T. (2014) A Facile Dedoping Approach for Effectively Tuning Thermoelectricity and Acidity of PEDOT: PSS Films. Organic Electronics, 15, 641-645. https://doi.org/10.1016/j.orgel.2013.12.023

[22] Park, H., Lee, S.H., Kim, F.S., Choi, H.H., Cheong, I.W. and Kim, J.H. (2014) Enhanced Thermoelectric Properties of PEDOT: PSS Nanofilms by a Chemical Dedoping Process. Journal of Materials Chemistry A, 2, 6532-6359. https://doi.org/10.1039/C3TA14960A

[23] Song, H., Kong, F., Liu, C., Xu, J., Jiang, Q. and Shi, H. (2013) Improved Thermoelectric Performance of PEDOT: PSS Film Treated with Camphorsulfonic Acid. Journal of Polymer Research, 20, 316-321. https://doi.org/10.1007/s10965-013-0316-0

[24] Kim, J., Jang, J.G., Hong, J.I., Kim, S.H. and Kwak, J. (2016) Sulfuric Acid Vapor Treatment for Enhancing the Thermoelectric Properties of PEDOT: PSS Thin-Films. Journal of Materials Science: Materials in Electronics, 27, 6122-6127. 
[25] Alemu, D., Wei, H.Y., Ho, K.C. and Chu, C.W. (2012) Highly Conductive PEDOT: PSS Electrode by Simple Film Treatment with Methanol for ITO-Free Polymer Solar Cells. Energy \& Environmental Science, 5, 9662-9671. https://doi.org/10.1039/c2ee22595f

[26] Wu, Y., Yi, Z., Ge, Z., Du, F. and Zhang, Y. (2017) The Latest Research Progress in Improving the Conductivity of PEDOT: PSS. Materials Guide A, 31, 26-31.

[27] Shi, H., Liu, C., Jiang, Q. and Xu, J. (2015) Effective Approaches to Improve the Electrical Conductivity of PEDOT: PSS: A Review. Advanced Electronic Materials, 1, 0282. https://doi.org/10.1002/aelm.201500017

[28] Kim, N., Lee, B.H., Choi, D., Kim, G., Kim, H., Kim, J.R., Lee, J., Kahng, Y.H. and Lee, K. (2012) Role of Lnterchain Coupling in the Metallic State of Conducting Polymers. Physical Review Letters, 109, Article ID: 106405.

[29] Zotti, G., Zecchin, S., Schiavon, G., Louwet, F., Groenendaal, L., Crispin, X., Osikowicz, W., Salaneck, W. and Fahlman, M. (2003) Electrochemical and XPS Studies toward the Role of Monomeric and Polymeric Sulfonate Counterions in the Synthesis, Composition, and Properties of Poly(3,4-ethylenedioxythiophene). Macromolecules, 36, 3337-3344. https://doi.org/10.1021/ma021715k

[30] Yu, C., Choi, K., Yin, L. and Grunlan, J.C. (2011) Light-Weight Flexible Carbon Nanotube Based Organic Composites with Large Thermoelectric Power Factors. ACS Nano, 5, 7885-7892. https://doi.org/10.1021/nn202868a

[31] Liu, G., Shi, F., Li, Y., Zhang, Q. and Wang, H. (2015) Preparation and Electrical Properties of Graphene Coated Glass Fiber Composites. Journal of Inorganic Materials, 30, 764-768.

[32] Zang, D., Liu, F., Zhang, M., Niu, X., Gao, Z. and Wang, C. (2015) Superhydrophobic Coating on Fiberglass Cloth for Selective Removal of Oil from Water. Chemical Engineering Journal, 262, 210-216. https://doi.org/10.1016/j.cej.2014.09.082

[33] Liu, Z., Zhang, Q., Wang, H. and Li, Y. (2013) Magnetic Field Induced Formation of Visually Structural Colored Fiber in Micro-Space. Journal of Colloid and Interface Science, 406, 18-23. https://doi.org/10.1016/j.jcis.2013.05.057

[34] Makyła, K., Müller, C., Lörcher, S., Winkler, T., Nussbaumer, M.G., Eder, M. and Bruns, N. (2013) Fluorescent Protein Senses and Reports Mechanical Damage in Glass-Fiber-Reinforced Polymer Composites. Advanced Materials, 25, 2701-2706. https://doi.org/10.1002/adma.201205226

[35] Faverolle, F., Attias, A.J., Bloch, B., Audebert, P. and Andrieux, C.P. (2007) Highly Conducting and Strongly Adhering Polypyrrole Coating Layers Deposited on Glass Substrates by a Chemical Process. Chemistry of Materials, 10, 740-752. https://doi.org/10.1021/cm970466p

[36] Guo, C., Duan, H., Dong, C., Zhao, G., Liu, Y. and Yang, Y. (2015) Preparation of the Polypropylene/Nickel Coated Glass Fibers Conductive Composites with a Low Percolation Threshold. Materials Letters, 143, 124-127. https://doi.org/10.1016/j.matlet.2014.12.091

[37] Guan, A., Wang, H., Jin, H., Chu, W., Guo, Y., and Lu, G. (2013) An Experimental Apparatus for Simultaneously Measuring Seebeck Coefficient and Electrical Resistivity from $100 \mathrm{~K}$ to $600 \mathrm{~K}$. Review of Scientific Instruments, 84, Article ID: 043903. https://doi.org/10.1063/1.4798647

[38] Chabinyc, M. (2014) Thermoelectric Polymers: Behind Organics' Thermopower. Nature Materials, 13, 119-121. https://doi.org/10.1038/nmat3859

[39] Bubnova, O., Khan, Z.U., Wang, H., Braun, S., Evans, D.R., Fabretto, M., Hoja- 
ti-Talemi, P., Dagnelund, D., Arlin, J.B., Crispin, X., et al. (2014) Semi-Metallic Polymers. Nature Materials, 13, 190-194. https://doi.org/10.1038/nmat3824

[40] Conwell, E.M. and Mizes, H.A. (1991) Metallic State of Polymers with Nondegenerate Ground States. Physical Review B Condensed Matter, 44, 937-942. https://doi.org/10.1103/PhysRevB.44.937

[41] Ando, K., Watanabe, S., Mooser, S., Saitoh, E. and Sirringhaus, H. (2013) Solution-Processed Organic Spin-Charge Converter. Nature Materials, 12, 622-627. https://doi.org/10.1038/nmat3634

[42] Liu, Y., Zhang, M., Ji, A., Yang, F. and Wang, X. (2016) Measuring Methods for Thermoelectric Properties of One-Dimensional Nanostructural Materials. RSC Advances, 6, 48933-48961. https://doi.org/10.1039/C5RA23634G 